OPEN ACCESS

Edited by:

Salvatore Salomone,

University of Catania, Italy

Reviewed by:

Ludovic Pelligand,

Royal Veterinary College (RVC), United Kingdom

Pierre-Louis Toutain,

Ecole Nationale Vétérinaire

de Toulouse, France

${ }^{*}$ Correspondence:

Yun Liu

abliuyun@yeah.net

Specialty section:

This article was submitted to Experimental Pharmacology and

Drug Discovery,

a section of the journal

Frontiers in Pharmacology

Received: 22 April 2019

Accepted: 26 August 2019

Published: 24 September 2019

Citation:

Yang Q, Liu X, Zhang C, Yong $K$, Clifton AC, Ding $H$ and

Liu Y (2019) Pharmacokinetics and Pharmacodynamics of

Gamithromycin Treatment of Pasteurella multocida in a Murine Lung Infection Model.

Front. Pharmacol. 10:1090. doi: 10.3389/fphar.2019.01090

\section{Pharmacokinetics and Pharmacodynamics of Gamithromycin Treatment of Pasteurella multocida in a Murine Lung Infection Model}

\author{
Qingwen Yang 1,2, Xuesong Liu', Chenghuan Zhang ${ }^{1}, K^{1}$ ang Yong ${ }^{2}$, Alancia Carol Clifton', \\ Huanzhong Ding ${ }^{3}$ and Yun Liu ${ }^{1 *}$ \\ ${ }^{1}$ Heilongjiang Key Laboratory for Laboratory Animals and Comparative Medicine, Department of Veterinary Surgery, College \\ of Veterinary Medicine, Northeast Agricultural University, Harbin, China, ${ }^{2}$ Laboratory of Veterinary Pharmacology, Department \\ of Animal Science and Technology, Chongqing Three Gorges Vocational College, Chongqing, China, ${ }^{3}$ Laboratory of \\ Veterinary Pharmacology, College of Veterinary Medicine, South China Agricultural University, Guangzhou, China
}

Gamithromycin is approved for the treatment and prevention of bovine respiratory disease (BRD), which is caused mainly by Mannheimia haemolytica, Pasteurella multocida, Histophilus somni, and Mycoplasma species. In this study, multiple dosage regimens were administered to the neutropenic mouse lung infection model in order to investigate the pharmacokinetic/pharmacodynamic (PK/PD) parameters of gamithromycin treatment of $P$. multocida and to further define the PK/PD parameter that best correlates with the efficacy of gamithromycin against $P$. multocida. The PK characteristics of gamithromycin were analyzed after a single subcutaneous (s.c.) injection (1, 3, 6, and $9 \mathrm{mg} / \mathrm{kg}$ ). The concentration-time profiles of unbound $(f)$ gamithromycin in plasma samples were analyzed by non-compartmental analysis. The main PK parameters of gamithromycin for the area under the concentration-time curve from 0 to $24 \mathrm{~h}\left(f \mathrm{AUC}_{0-24}\right)$ and the peak drug concentration $\left(f C_{\max }\right.$ ) values ranged from 0.86 to $8.42 \mu \mathrm{g} \cdot \mathrm{h} / \mathrm{ml}$ and from 0.55 to $5.69 \mu \mathrm{g} / \mathrm{ml}$, respectively. The PD values were calculated based on multiple s.c. injections over $24 \mathrm{~h}$ $(1,3,6$, and $9 \mathrm{mg} / \mathrm{kg}$ at $6,8,12$, and $24 \mathrm{~h}$, respectively; total dosage 1-36 mg/ $\mathrm{kg}$ ). The minimum inhibitory concentration (MIC) of gamithromycin against $P$. multocida in mice serum was $0.15 \mu \mathrm{g} / \mathrm{ml}$. Analysis of PK/PD indices using the inhibitory effect $E_{\max }$ model indicated a strong correlation $\left(R^{2}=0.9624\right)$ between the $f \mathrm{AUC}_{0-24} / \mathrm{MIC}$ ratio and various antibacterial effects. The area under the unbound concentration-time curve over $24 \mathrm{~h}$ to $\mathrm{MIC}$ ( $f \mathrm{AUC}_{0-24} / \mathrm{MIC}$ ) predicted for bacteriostatic action, 1- $\log _{10}$ reduction, 2- $\log _{10}$ reduction, and $3-\log _{10}$ reduction were $56.77,90.18,143.06$, and $239.44 \mathrm{~h}$, respectively. These in vivo data may facilitate gamithromycin dosage optimization against $P$. multocida in veterinary medicine.

Keywords: gamithromycin, lung infection model, Pasteurella multocida, mice, pharmacokinetic/pharmacodynamic 


\section{INTRODUCTION}

Bovine respiratory disease (BRD), which is one of the most common respiratory diseases in calves, occurs with high mortality and morbidity rates, leading to enormous economic losses in the cattle industry. The risk factors involved in BRD are diverse and complex, including mixing, transportation, viral and bacterial agents, natural immune responses, and others (Snowder et al., 2006; Dabo et al., 2007; Jones and Chowdhury, 2007; Snowder et al., 2007; Griffin et al., 2010). Although the pathogenesis of BRD is multifactorial, pathogenic bacteria, such as Mannheimia haemolytica, Pasteurella multocida, Histophilus somni, and Mycoplasma species, are the main contributing factors which contribute to morbidity and mortality (Portis et al., 2012).

Gamithromycin, a novel semi-synthetic macrolide, is approved for the treatment and prevention of BRD (Muraro et al., 2012; O'Connor et al., 2016). Like other macrolides, it plays a bacteriostatic and bactericidal role by inhibiting the ribosomal 50S subunit. Prior studies have demonstrated that gammamycin has high antibacterial activity against Mycoplasma mycoides, M. haemolytica, and P. multocida (Rossi et al., 2010; Baggott et al., 2011; Forbes et al., 2011; Mitchell et al., 2013; Abell et al., 2017; Snyder et al., 2017). Pharmacokinetic (PK) data for gamithromycin delivered by subcutaneous (s.c.) injection have been reported in different species, such as foals, sheep, cattle, broiler chickens, and pigs (Watteyn et al., 2013; Kellermann et al., 2014; Wyns et al., 2014; Jones et al., 2015; Berlin et al., 2017).

Selecting effective PK/pharmacodynamic (PD) indices for analysis is crucial for optimization of the dose regimens in veterinary medicine (Toutain et al., 2002). PK/PD of gamithromycin has been investigated in cattle and turkey poults (Dedonder et al., 2015; Watteyn et al., 2015). Previous study has demonstrated that higher PK/PD indices showed the strongest correlation to positive treatment outcomes (Dedonder et al., 2015). However, there are no reports of the in vivo $\mathrm{PK}$ data combined with in vivo $\mathrm{PD}$ for the evaluation of the antibacterial activity of gamithromycin against $P$. multocida using a neutropenic murine lung infection model, which is commonly used to investigate the relationship between host, drug, and pathogens (Andes and Craig, 2006; Ferran et al., 2011; Qu et al., 2015; So et al., 2015; Thabit et al., 2016; Zhou et al., 2017b; Zeng et al., 2018). This relatively stable and mature model avoids the impact of the host immune system on antimicrobial efficacy. Using this model for analysis of the in vivo changes in unbound drug concentration in plasma indicated that changes in the amount of bacteria were highly related to the antibacterial activity of the drug. For macrolides, the neutropenic mouse lung infection model was used to evaluate the antibacterial activity of tildipirosin against $P$. multocida (Zeng et al., 2018).

In this study, we investigated the PK and PD characteristics of gamithromycin against $P$. multocida in a neutropenic murine lung infection model and determined the value of PK/PD index to achieve various antibacterial activity.

\section{MATERIALS AND METHODS}

\section{Antibiotics and Bacteria}

Gamithromycin standard (purity 98.6\%) and d5-gamithromycin (purity: 93.7\%) was provided by Zhong sheng tiao zhan Biotechnology, Co., Ltd (Tianjin, China). Cyclophosphamide was purchased from Aladdin, Co., Ltd (Shanghai, China). ICR mice plasma was purchased from Ruite Biotechnology, Co., Ltd (Guangzhou, China). P. multocida NM-5-7, isolated from a yellow cattle which died of hemorrhagic septicemia in Neimenggu province, was identified by matrix-assisted laser desorption/ionization time-of-flight (MALDI-TOF)/mass spectrometry (MS) analysis (AXIMA Assurance, Shimadzu). Six clinical strains of P. multocida (NU01-NU06) were provided by the Department of Preventive Veterinary Medicine, College of Veterinary Medicine, Northeast Agricultural University.

\section{Animals}

Specific-pathogen-free (SPF) female ICR mice (aged 6 weeks; 33-37 g) were purchased from Guangdong Medical Lab Animal Center (Guangzhou, China). Mice were maintained with SPF food and water for 1 week.

\section{In Vitro Susceptibility Studies}

The minimum inhibitory concentration (MIC) of gamithromycin against $P$. multocida was determined in serum and MuellerHinton broth (MHB) using Clinical and Laboratory Standards Institute microdilution methods. Briefly, a minimum of 10 freshly cultured colonies were transferred into MHB and incubated at $37^{\circ} \mathrm{C}$ on a shaking incubator $(220 \mathrm{rpm})$ for $5 \mathrm{~h}$ [approximately $8 \mathrm{log}$ colony-forming unit $(\mathrm{CFU}) / \mathrm{ml}$. Culture aliquots of $100 \mu \mathrm{l}$ were added into a 96-well plate to obtain a series of twofold-dilution drug concentrations. Three overlapping sets of doubling dilutions were used to improve the accuracy of the MIC determinations $(0.025-0.8,0.031-1$, and $0.038-1.2 \mu \mathrm{g} / \mathrm{ml})$. The MIC was defined as the lowest concentration of gamithromycin that inhibited the visible bacterial growth in serum and MHB. Susceptibility testing was performed in triplicate, and Staphylococcus aureus ATCC 29213 was used as control strain.

\section{Neutropenic Mouse Lung Infection Model}

The neutropenic mouse lung infection model was established as previously described (Ferran et al., 2011; Qu et al., 2015; Zeng et al., 2018). Briefly, neutropenic mice were successfully produced (neutrophil count $<100 \mathrm{~mm}^{3}$ ) after intraperitoneal cyclophosphamide injection $(150 \mathrm{mg} / \mathrm{kg}$ daily for 4 days followed by one dose at $100 \mathrm{mg} / \mathrm{kg}$ on day 5). Lung infection was achieved by tracheal administration of $P$. multocida NM-5-7. Following anesthesia with pentobarbital sodium, an intravenous catheter (22 G, "Y" type, without mandrel) was inserted into the trachea of neutropenic mice, and $50 \mu \mathrm{l}$ of bacterial suspension (approximately $8 \log _{10} \mathrm{CFU} / \mathrm{ml}$ ) was delivered. The mice were then placed in an inverted position for approximately $15 \mathrm{~s}$. The neutropenic mouse lung infection model was established, when the bacterial burden reached $6 \log _{10} \mathrm{CFU}$ per lung. 


\section{PK Analysis of Gamithromycin in Neutropenic Lung Infected Mice}

Approximately $3 \mathrm{~h}$ after bacterial inoculation, when the bacterial burden was $6.12 \pm 0.08 \log _{10}$ CFU per lung, $200 \mu \mathrm{l}$ of gamithromycin was administered using single s.c. doses of 1 , 3,6 , and $9 \mathrm{mg} / \mathrm{kg}$. Sedation management was administered as previously described (Zeng et al., 2018). Briefly, the mice were placed in an induction chamber with an oxygen flow rate of $0.5-$ $1.0 \mathrm{~L} / \mathrm{min}$. Isoflurane vapor (3-5\%) was applied for induction and then reduced (1-3\%) for maintenance. Retro-orbital blood samples were collected into $1.5-\mathrm{ml}$ plastic tubes (containing heparin sodium) at $0.083,0.167,0.25,0.5,0.75,1,2,3,6,9,12$, and $24 \mathrm{~h}$ after treatment $(n=5)$. Each mouse was sampled at three or four time points. This was decided based on a tradeoff between reducing inter-animal variability to obtain accurate PK data, reducing the number of mice used and to adhere to acceptable welfare conditions. Plasma was isolated following centrifugation $\left(4,500 \times g\right.$ for $10 \mathrm{~min}$ at $\left.4^{\circ} \mathrm{C}\right)$ and stored at $-80^{\circ} \mathrm{C}$ for analysis.

Plasma concentrations of gamithromycin were analyzed as previously described (Dedonder et al., 2015). Briefly, $100 \mu \mathrm{l}$ of each plasma sample was added to $10 \mu \mathrm{l}$ of d5-gamithromycin $(1 \mu \mathrm{g} / \mathrm{ml})$ and $400 \mu \mathrm{l}$ of formic acid (1\% in acetonitrile) and then vortexed for $1 \mathrm{~min}$. After centrifugation $(3,500 \times g$ for $10 \mathrm{~min}$ at $4^{\circ} \mathrm{C}$ ), $200 \mu \mathrm{l}$ of supernatant was diluted with 600 $\mu \mathrm{l}$ of ultrapure water for liquid chromatography-tandem mass spectrometry (LC-MS/MS) analysis of gamithromycin concentrations by extrapolation against a standard calibration curve $(0.01-4 \mu \mathrm{g} / \mathrm{ml})$. The limit of detection (LOD) and limit of quantification (LOQ) values for this assay were 0.005 and $0.01 \mu \mathrm{g} / \mathrm{ml}$, respectively. The recoveries of gamithromycin in plasma samples were $>85 \%$. All inter- and intra-assay variations measured by calculation of relative standard deviation (\%RSD) were $<10 \%$.

Plasma PK parameters of gamithromycin, such as the elimination half-life $\left(t_{1 / 2}\right)$, the time of maximum concentration $\left(T_{\max }\right)$, the area under the concentration-time curve from 0 to $24 \mathrm{~h}\left(f \mathrm{AUC}_{0-24}\right)$, and the peak drug concentration $\left(f \mathrm{C}_{\max }\right)$, were analyzed by non-compartmental analysis (WinNonlin 5.2.1, Pharsight Corporation, Mountain View, CA, USA). PK parameters were represented as means \pm standard deviation (SD).

\section{In Vitro Binding of Gamithromycin to Plasma Proteins}

The protein binding in mice plasma was determined by ultrafiltration methods as previously described (Huang et al., 2010). Briefly, plasma was spiked with appropriate amounts of gamithromycin to achieve final concentrations of $0.01,0.4$, 1 , and $4 \mu \mathrm{g} / \mathrm{ml}$ and then vortexed and incubated at $35 \pm 2{ }^{\circ} \mathrm{C}$ for $30 \mathrm{~min}$. Aliquots of $1 \mathrm{ml}$ of the plasma mixtures were then transferred to Millipore Centrifree ultrafiltration devices and centrifuged at approximately $1,730 \times g$ for $30 \mathrm{~min}$ at $20^{\circ} \mathrm{C}$. Subsequently, $10 \mu \mathrm{l}$ of $\mathrm{d} 5$-gamithromycin $(1 \mu \mathrm{g} / \mathrm{ml})$ was added to $100 \mu \mathrm{l}$ of each filtrate and determined using the above-mentioned method. Plasma mixtures of $100 \mu \mathrm{l}$ without ultrafiltration procedures were also determined using the same method. Three separate sets of experiments were undertaken for each concentration. The drug percentage bounded to protein was calculated as follows:

$$
\% \text { Bound }=\left(\frac{\text { concentration in plasma }- \text { concentration in filtrate }}{\text { concentration in plasma }}\right) \times 100
$$

\section{PD Study in the Neutropenic Mouse Lung Infection Model}

Following the successful establishment of the neutropenic lung infection model, mice were divided randomly into 16 experimental groups ( $n=3$ per group). The experimental groups were treated with gamithromycin via s.c. injection $3 \mathrm{~h}$ after P. multocida NM-5-7 inoculation; this was designated as the 0 -h time point for treatment. The dosing regimens were $1,3,6$, and $9 \mathrm{mg} / \mathrm{kg}$ every $6,8,12$, and $24 \mathrm{~h}$, respectively. The range of gamithromycin for this study was from 1 to $36 \mathrm{mg} / \mathrm{kg}$ per $24 \mathrm{~h}$. All mice in experimental groups were euthanized by $\mathrm{CO}_{2}$ asphyxiation $24 \mathrm{~h}$ after treatment. Mice in the untreated control group were sacrificed prior to the 0 -h time point for gamithromycin treatment and $24 \mathrm{~h}$ after treatment $(n=3$ per time point). The CFU in each homogenized lung sample was determined after sacrifice as previously described (Qu et al., 2015). Briefly, all lung samples were aseptically removed and homogenized in sterilized saline $(5 \mathrm{ml})$. The homogenates were then diluted (10-fold serial dilutions). Twenty microliters of each lung homogenate dilution was dropped onto $5 \%$ sterile defibrinated sheep blood trypticase soy agar (5\% BTSA). The homogenate dilution was dropped in triplicate. Finally, the bacterial colonies were counted on the 5\% BTSAs after incubation for $18-24 \mathrm{~h}$ at $37^{\circ} \mathrm{C}$, and the mean values were used for data analysis. The LOD was $50 \mathrm{CFU} / \mathrm{ml}$.

\section{PK/PD Integration and Modeling}

The PK parameters of gamithromycin were calculated using WinNonlin software version 5.2.1 (Pharsight Corporation), after single s.c. injections of $1,3,6$, and $9 \mathrm{mg} / \mathrm{kg}$. The noncompartmental analysis was performed, and the extravascular input best reflected the unbound plasma concentrationtime profiles of gamithromycin. The PK/PD indices were calculated using the inhibitory effect $I_{\max }$ model according to the following equation:

$$
E=E_{0}-\frac{I_{\max } \times X}{I C_{50}+X}
$$

where $E$ represents the antibacterial effect, defined as the change between the treated mice after $24 \mathrm{~h}$ and the initial untreated control mice; $I_{\max }$ denotes the maximum change in $\log _{10}$ CFU per lung after 24-h treatment with gamithromycin; $E_{0}$ indicates the change in $\log _{10} \mathrm{CFU}$ per lung in control samples between time 0 and $24 \mathrm{~h} ; I C_{50}$ represents the $X$ value producing $50 \%$ of the $I_{\max }$, where $X$ indicates the PK/PD index (e.g. $f \mathrm{AUC}_{0-24} / \mathrm{MIC}, f C_{\max } /$ $\mathrm{MIC}$, and \%T > MIC). PD indices were analyzed by non-linear regression using WinNonlin software. Due to regression in each of the PK/PD indices, in addition to $R^{2}$, the percentage of the variance explained by the model is given by $R^{2}$. 


\section{Statistical Analyses}

Statistical analyses were done by using analysis of variance, and significant differences were analyzed using Bonferroni's correction for inter-group comparisons. Differences were considered significant when $P<0.05$.

\section{RESULTS}

\section{In Vitro Susceptibility Testing}

The MICs of gamithromycin against $P$. multocida in MHB and serum are shown in Table 1. The average MICs in MHB, mouse serum, and adult bovine serum were $0.71,0.107$, and $0.036 \mu \mathrm{g} / \mathrm{ml}$, respectively. The average MIC in mouse serum was threefold higher than that in adult bovine serum.

\section{In Vitro Binding of Gamithromycin to Plasma Proteins}

The protein binding of gamithromycin in mouse plasma with a concentration range of $0.01-4 \mu \mathrm{g} / \mathrm{ml}$ is shown in Table 2 . The average binding was found to be $27.2 \pm 1.8 \%$.

\section{PK of Gamithromycin in Neutropenic Lung Infected Mice}

The unbound plasma gamithromycin concentration-time profiles in neutropenic lung infected mice after single s.c. injection at 1, 3, 6, and $9 \mathrm{mg} / \mathrm{kg}$ are presented in Figure 1. Unbound plasma gamithromycin concentrations were calculated based on a plasma protein binding activity of $27.2 \%$. The main PK parameters are shown in Table 3. The $f \mathrm{AUC}_{0-24}$ and $f \mathrm{C}_{\max }$ values ranged from 0.86 to $8.42 \mu \mathrm{g} \cdot \mathrm{h} / \mathrm{ml}$ and 0.55 to $5.69 \mu \mathrm{g} / \mathrm{ml}$, respectively. Both the $f \mathrm{AUC}_{0-24}$ and $f C_{\max }$ values increased with dose escalation (1-9 mg/kg). Moreover, the $t_{1 / 2}$ values ranged from 8.04 to $13.64 \mathrm{~h}$.

\section{PK/PD Integration and Modeling}

Gamithromycin was administered when the bacterial load reached $6.12 \pm 0.08 \log _{10} \mathrm{CFU} / \mathrm{lung}$ approximately $3 \mathrm{~h}$ after inoculation. In the untreated control group, the bacterial load reached $8.91 \pm 0.03 \log _{10}$ CFU per lung across the $24-\mathrm{h}$ treatment period. The maximum antibacterial effect in experimental groups was reduced to a rate of $2.33 \pm 0.05 \log _{10} \mathrm{CFU}$ per lung $24 \mathrm{~h}$ after treatment. The fractionated dose regimen curves were consistent, indicating that the $f \mathrm{AUC}_{0-24} /$ $\mathrm{MIC}$ ratio is the predictive $\mathrm{PD}$ index (Figure 2). The main $\mathrm{PK} / \mathrm{PD}$ indices are shown in Table 4. In this model, the $f \mathrm{AUC}_{0-24} / \mathrm{MIC}$ ratio was regarded as the best $\mathrm{PK} / \mathrm{PD}$ index of the antibacterial effect $\left(R^{2}=0.9624\right)$. Correlations between PK/PD indices and antibacterial effects are presented in Figures 3 and 4.

\section{DISCUSSION}

PK studies of gamithromycin in many different animals, including foals, cattle, broiler chickens, sheep, and pigs indicate that gamithromycin shares characteristics with macrolides, including rapid absorption, wide distribution, and prolonged halflife (Watteyn et al., 2013; Kellermann et al., 2014; Wyns et al., 2014; Jones et al., 2015; Berlin et al., 2017). In this present study, the $T_{\max }$ of plasma gamithromycin was $0.17 \mathrm{~h}$ in mice after a single s.c. injection at a recommended dosage of $6 \mathrm{mg} / \mathrm{kg}$, which was higher than values obtained using the same dosage regimen in cattle $(1 \mathrm{~h})$, sheep $(0.911 \mathrm{~h})$, and pigs (0.63 h) (Huang et al., 2010; Kellermann et al., 2014; Wyns et al., 2014). Slightly higher values were obtained in broiler chickens $(0.13 \mathrm{~h})$ (Watteyn et al., 2013). Inter-species differences might be the major reason for these different outcomes. In the current study, both the $f \mathrm{AUC}_{0-24}$ and $f C_{\max }$ values were proportional to the dose, similar results were also previously reported in neutropenic lung infected mice model for tildipirosin against $P$. multocida (Zeng et al., 2018). Moreover, the $f \mathrm{AUC}_{0-24}$ and $f C_{\max }$ values $6.33 \mu \mathrm{g} \cdot \mathrm{h} / \mathrm{ml}$ and $3.4 \mu \mathrm{g} / \mathrm{ml}$ respectively, were higher than those obtained in tildipirosin against $P$. multocida, after using the same dosage at $6 \mathrm{mg} / \mathrm{kg}$.

The combination of $\mathrm{PK}$ and $\mathrm{PD}$ used in predicting the antimicrobial activity of antimicrobial agents and calculating the dosage of antibiotics to achieve different antimicrobial efficacy is regarded as a very useful method to optimize the dosage regimen in veterinary clinical settings (Toutain et al., 2002; Lees et al., 2006). Therefore, it is very important to select effective PK/PD indices for optimization of the dose regimens. Macrolides are usually classified as time-dependent antimicrobial agents. In a previous study, the $\mathrm{PK} / \mathrm{PD}$ index $\% T>\mathrm{MIC}$ showed the strongest correlation with antibacterial activity (Ahmad et al., 2016). However, for the second generation of macrolides, including tulathromycin and tildipirosin, the $f \mathrm{AUC}_{0-24} / \mathrm{MIC}$ ratio has been reported as the best $\mathrm{PK} / \mathrm{PD}$ index used to describe the antibacterial effect against $P$. multocida (Zhou et al., 2017a; Zeng et al., 2018). A previous study investigated tulathromycin against $P$. multocida in a porcine tissue cage model; results indicated that $\mathrm{AUC}_{0-24} / \mathrm{MIC}$ exhibited a high

TABLE 1 | The minimum inhibitory concentration (MIC) of gamithromycin against Pasteurella multocida in Mueller-Hinton broth (MHB) and serum.

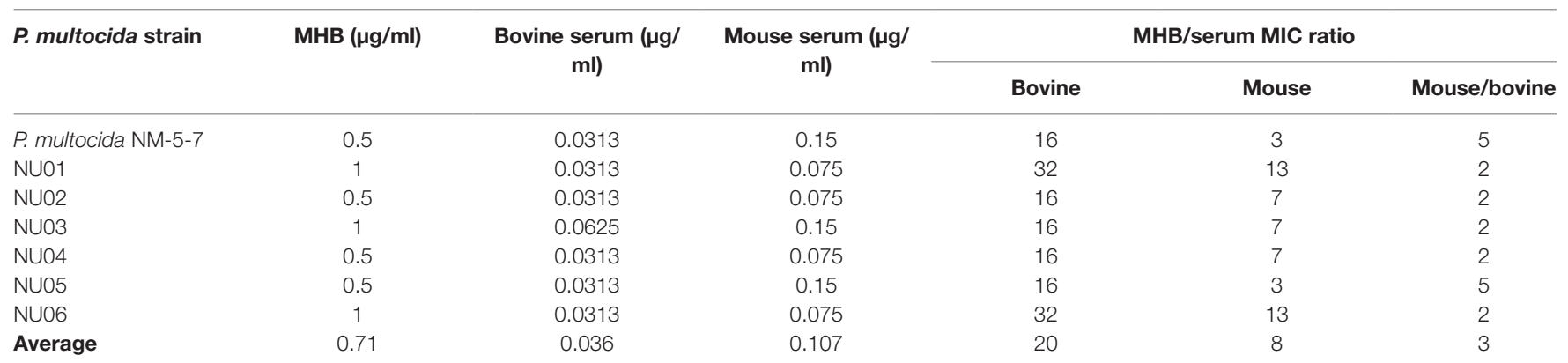


TABLE 2 | Percentage of bound gamithromycin in plasma of mice.

\begin{tabular}{|c|c|c|c|c|}
\hline Concentration $(\mu \mathrm{g} / \mathrm{ml})$ & Lot 1 & Lot 2 & Lot 3 & Average $^{(\mathrm{A})}$ \\
\hline 0.01 & 26.5 & 29.8 & 27.2 & $27.8 \pm 1.4$ \\
\hline 0.04 & 23.8 & 22.5 & 29.5 & $25.3 \pm 3.0$ \\
\hline 1 & 25.8 & 27.9 & 23.6 & $25.8 \pm 1.8$ \\
\hline 4 & 31.2 & 30.3 & 27.7 & $29.7 \pm 1.5$ \\
\hline Average $^{(\mathrm{B})}$ & $26.8 \pm 2.7$ & $27.6 \pm 3.1$ & $27.0 \pm 2.1$ & $27.2 \pm 1.8^{(\mathrm{C})}$ \\
\hline
\end{tabular}

(A) Numbers are the average \pm standard deviation of three lots of plasma.

(B) Numbers are the average \pm standard deviation of four concentrations.

(C) Value is the average \pm standard deviation of the four concentrations from the average of the three lots.

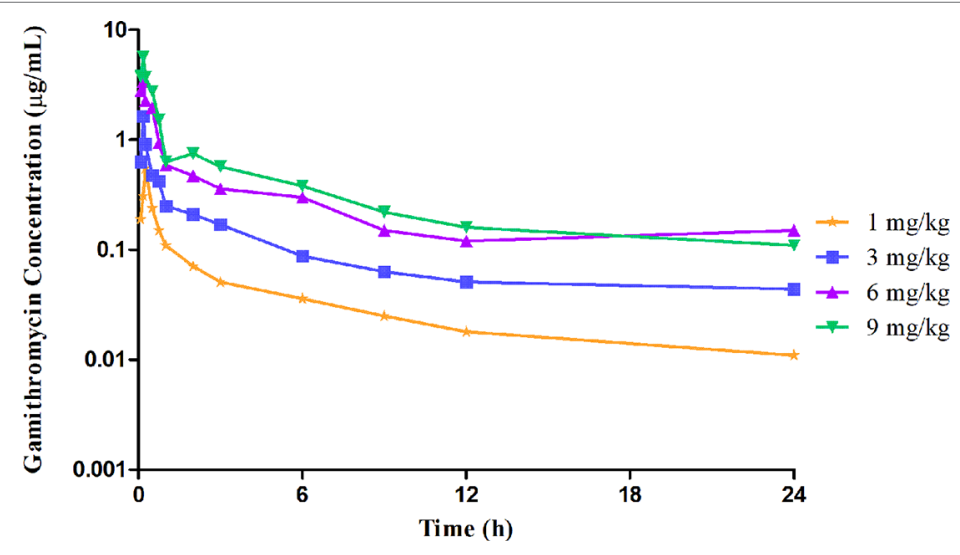

FIGURE 1 | Unbound plasma gamithromycin concentration-time courses in neutropenic infected mice following single subcutaneous injections of $1,3,6$, and 9 $\mathrm{mg} / \mathrm{kg}$.

TABLE 3 | The pharmacokinetic parameters of gamithromycin in neutropenic lung infected mice plasma after single subcutaneous (s.c.) injection dose levels at $1,3,6$, and $9 \mathrm{mg} / \mathrm{kg}$ of body weight.

\begin{tabular}{|c|c|c|c|c|}
\hline \multirow[t]{2}{*}{ Parameters (units) } & \multicolumn{4}{|c|}{ Dose levels } \\
\hline & $1 \mathrm{mg} / \mathrm{kg}$ & $3 \mathrm{mg} / \mathrm{kg}$ & $6 \mathrm{mg} / \mathrm{kg}$ & $9 \mathrm{mg} / \mathrm{kg}$ \\
\hline$f \mathrm{t}_{1 / 2}(\mathrm{~h})$ & 13.64 & 9.07 & 9.43 & 8.04 \\
\hline$T_{\max }(\mathrm{h})$ & 0.25 & 0.17 & 0.17 & 0.17 \\
\hline$f C_{\max }(\mu \mathrm{g} / \mathrm{ml})$ & 0.55 & 1.65 & 3.4 & 5.69 \\
\hline$f \mathrm{~A} \mathrm{C}_{0-24}(\mu \mathrm{g} \cdot \mathrm{h} / \mathrm{ml})$ & 0.86 & 2.37 & 6.33 & 8.42 \\
\hline
\end{tabular}

$f C_{\max }$ maximum unbound gamithromycin concentration in plasma; $T_{\max }$, time to achieve maximum unbound gamithromycin concentration in plasma; $f t_{1 / 2}$, elimination half-life; $f \mathrm{AUC}_{0-24}$, area under the unbound plasma gamithromycin concentrationtime curve over $24 \mathrm{~h}$.

correlation with the antibacterial effect in serum and the values of $\mathrm{AUC}_{0-24} / \mathrm{MIC}$ for bacteriostatic, 3- $\log _{10}$ reduction, and 4- $\log _{10}$ reduction were 44.55, 73.19, and $92.44 \mathrm{~h}$, respectively (Zhou et al., 2017a). Another study explored tildipirosin against $P$. multocida in a neutropenic lung infected mice model, suggesting that the respective values for bacteriostatic action, $1-\log _{10}$ reduction, and $2-\log _{10}$ reduction were achieved when $f \mathrm{AUC}_{0-24} / \mathrm{MIC}$ reached 19.93, 31.89, and 53.27 h, respectively (Zeng et al., 2018). These previous studies highlighted that the second generation of macrolides had a strong antimicrobial activity against $P$. multocida. However, for one of the second generation of macrolides, the efficacy of gamithromycin against $P$. multocida has not been studied. Therefore, a neutropenic lung infected mice model was used to evaluate the efficacy of gamithromycin against $P$. multocida.

In the current study, gamithromycin dose fractionation experiments during the 24-h treatment period showed that the correlation indices ( $R^{2}$ values) of the $f \mathrm{AUC}_{0-24} / \mathrm{MIC}$, the $f C_{\max } / \mathrm{MIC}$, and the $\% T>$ MIC were $0.9624,0.9424$, and 0.8725 , respectively. Based on the $R^{2}$ values, the $f \mathrm{AUC}_{0-24} / \mathrm{MIC}$ ratio was more predictive of the antimicrobial effect, which was similar to previous studies. The current study suggested that the $f \mathrm{AUC}_{0-24} / \mathrm{MIC}$ ratios required for bacteriostatic action, $1-\log _{10}$ reduction, and $2-\log _{10}$ reduction were $56.77,90.18$, and $143.06 \mathrm{~h}$, respectively, which were higher than estimated in a prior study (Zeng et al., 2018). Different drug and different strains of MIC might contribute to these variances.

Previous studies have shown that MICs in MHB differ from those in biological sample matrices, indicating that MICs in MHB were artificially high (Zhou et al., 2017a; Zeng et al., 2018). Thus, it is important to use the MIC obtained in biological sample matrices rather than in $\mathrm{MHB}$, as this may be considered more suitable for macrolide PK/PD integration (Lees et al., 2006; Toutain et al., 2017). To investigate the effect of serum on the determination of MICs, we determined the MICs of $P$. multocida NM-5-7 and six other P. multocida strains in serum and in MHB. The results showed that MICs of $P$. multocida were 26-fold lower in adult bovine serum than in $\mathrm{MHB}$, when we take the protein binding ratio of $26 \%$ into account. Compared with other strains 


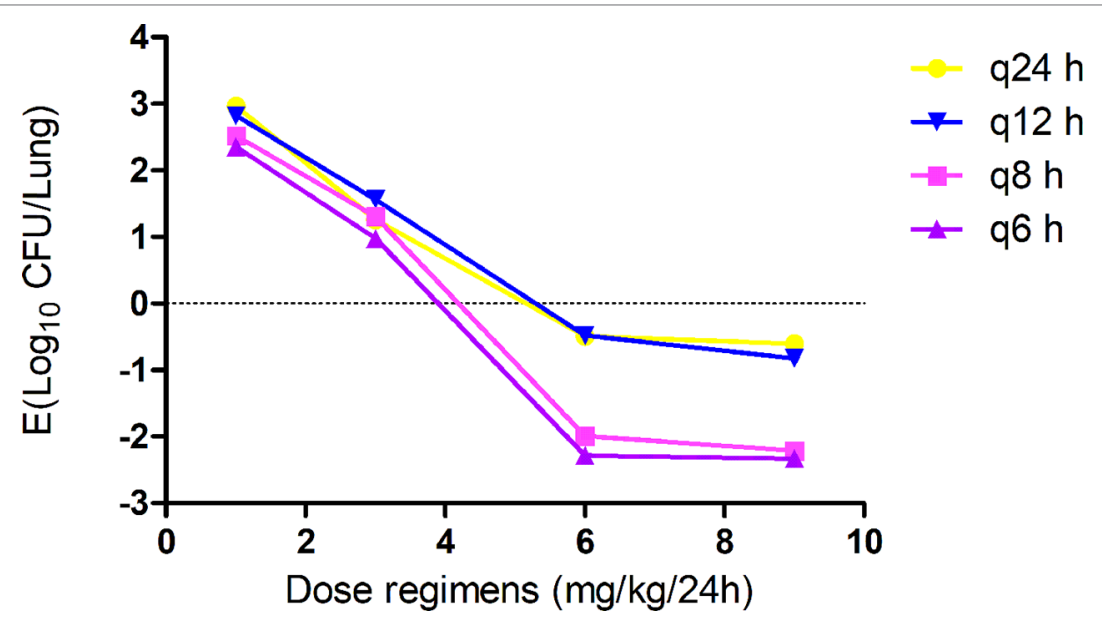

FIGURE 2 | In vivo dose fractionation with gamithromycin using the murine neutropenic lung infection model. Each symbol represents the mean value of lung bacterial infection from three mice infected with Pasteurella multocida NM-5-7. Sixteen dose regimens of gamithromycin were used to treat the P. multocida NM-5-7 infection. The change in the $\log _{10}$ number of colony-forming unit (CFU)/lung was measured at the start and after $24 \mathrm{~h}$ of therapy. Data points below the horizontal dashed line represent killing, and points above the horizontal dashed line represent growth.

TABLE 4 | The pharmacokinetic/pharmacodynamic (PK/PD) parameter estimates for the $f \mathrm{AUC}_{0-24} / \mathrm{MIC}$ to achieve various antibacterial effects.

\begin{tabular}{|c|c|}
\hline Parameters (units) & Values \\
\hline$E_{0}\left(\log _{10}\right.$ CFU/lung $)$ & 3.38 \\
\hline$I_{\max }\left(\log _{10}\right.$ CFU/lung $)$ & 8.81 \\
\hline $1 C_{50}(\mathrm{~h})$ & 91.25 \\
\hline$f \mathrm{AUC}_{0-24} / \mathrm{MIC}$ for bacteriostatic (h) & 56.77 \\
\hline$f \mathrm{AUC}_{0-24} / \mathrm{MIC}$ for $1-\log _{10}$ reduction (h) & 90.18 \\
\hline$f A \cup C_{0-24} / \mathrm{MIC}$ for $2-\log _{10}$ reduction (h) & 143.06 \\
\hline$f \mathrm{AUC}_{0-24} / \mathrm{MIC}$ for $3-\log _{10}$ reduction (h) & 239.44 \\
\hline
\end{tabular}

$I_{\text {max }}$ denotes the maximum change in $\log _{10}$ CFU/lung after 24- $h$ treatment with gamithromycin; $E_{0}$ indicates the change in $\log _{10}$ CFU/lung in control samples between time 0 and $24 \mathrm{~h} ; I_{50}$ represents the $f A U C_{0-24} \mathrm{~h} / \mathrm{MIC}$ value producing $50 \%$ of the $I_{\text {max }}$ AUC, area under the curve; CFU, colony-forming units; MIC, minimum inhibitory concentration. and macrolides, the MIC ratio of gamithromycin against $P$. multocida was much lower than that of gamithromycin against M. mycoides (Mitchell et al., 2013) and that of tulathromycin against P. multocida (Toutain et al., 2017). The susceptibility of different strains to different drugs leads to these results.

In the area of human medicine, the neutropenic mouse model is commonly used to predict the therapeutic dose for macrolides (Tessier et al., 2002; Hoffman et al., 2003). Thus, in the current study, we could extend to predict a daily dosage in cattle using the following equation: Dose $=\left[\mathrm{Cl} \times\left(\mathrm{AUC}_{0-24} / \mathrm{MIC}\right) \times \mathrm{MIC}_{90}\right] /(F \times$ fu) (Toutain et al., 2002). Combining the current data and previous data on cattle reported by Huang et al. $(\mathrm{Cl}=712 \mathrm{ml} / \mathrm{kg} / \mathrm{h}, \mathrm{fu}=0.74$, and $F=1$ ), daily dosages to achieve bacteriostatic effect, $1-\log _{10}$ reduction, $2-\log _{10}$ reduction, and $3-\log _{10}$ reduction were $2.10,3.34$,

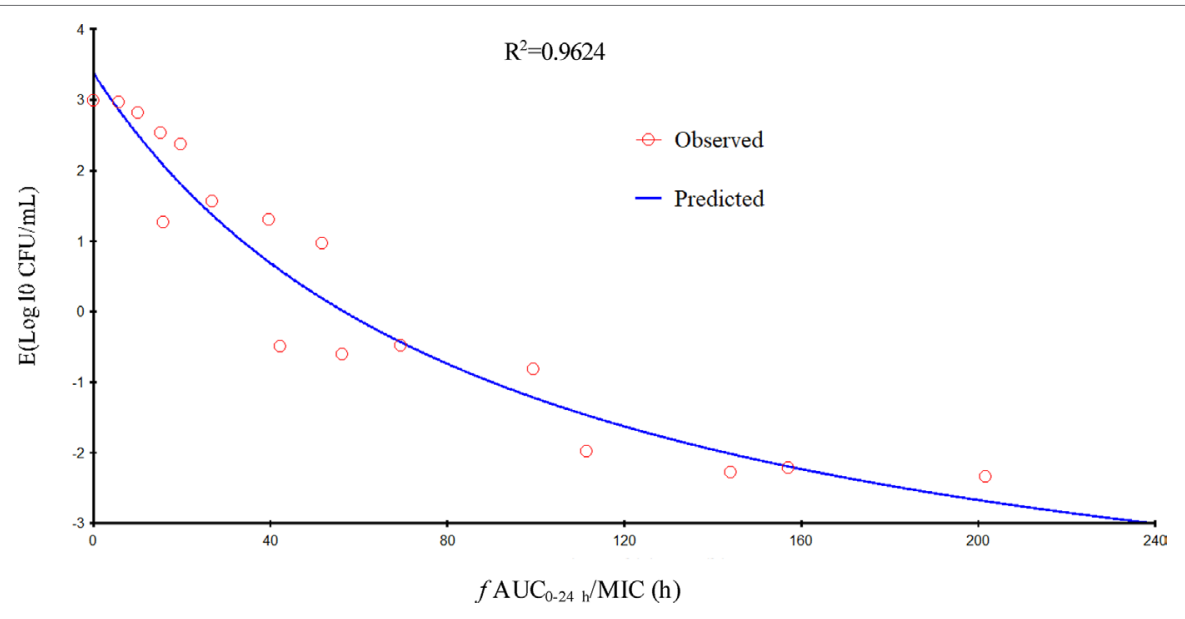

FIGURE 3 | Relationship of $f \mathrm{AUC}_{24} /$ minimum inhibitory concentration (MIC) for Pasteurella multocida NM-5-7 with the change in the log 10 number of colony-forming unit (CFU)/lung after $24 \mathrm{~h}$ of therapy. $R^{2}$ is the correlation coefficient. 


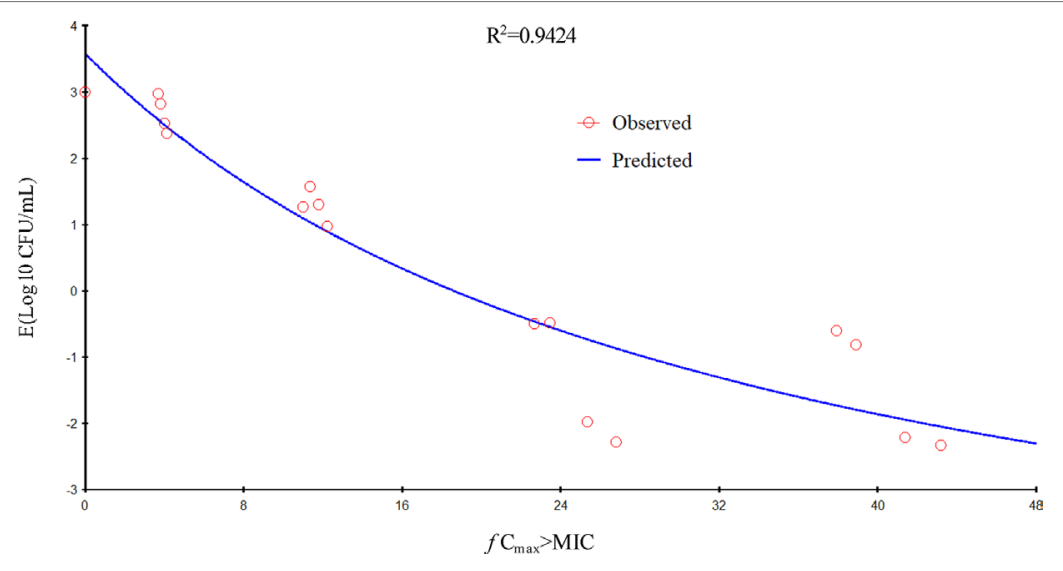

FIGURE 4 | Relationship of $f \mathrm{C}_{\text {max }} /$ minimum inhibitory concentration (MIC) for Pasteurella multocida NM-5-7 with the change in the log ${ }_{10}$ number of colony-forming unit (CFU)/lung after $24 \mathrm{~h}$ of therapy. $R^{2}$ is the correlation coefficient.

5.29 , and $8.86 \mathrm{mg} / \mathrm{kg}$, respectively. When we consider the effect of serum, more realistic doses were predicted for currently observed MICs in cattle, indicating that the MIC obtained in bovine serum was more suitable for macrolide PK/PD integration for cattle. Some evidence could be found in previous studies, which confirmed that the presence of natural media, such as serum and plasma, could reduce the MIC of bacteria by altering the integrity of gramnegative bacterial outer membrane and increasing outer-membrane permeability, thus increasing the accumulation of macrolides (Buyck et al., 2012; Mustafa et al., 2017). Obviously, the daily dosage (8.86 $\mathrm{mg} / \mathrm{kg}$ ) required for $3-\log _{10}$ reduction was much higher than the recommended dose $(6 \mathrm{mg} / \mathrm{kg})$, indicating that the recommended dose would fail to achieve an eradication effect. However, for longacting injection, a dose required for $2-\log _{10}$ reduction could be enough to reduce the incidence of disease (Toutain et al., 2017). Therefore, the recommended dose of gamithromycin would be effective in the treatment of $P$. multocida infection in veterinary clinical medicine. But, it is still worth remembering that these theoretical values still need validation in clinical practice.

A few limitations of this model should be noted. First, the clinical characteristics of laboratory infections differ from those of the natural infection. In the present study, only P. multocida was used for modeling, ignoring the clinical influences of other possible bacterial pathogens ( $M$. haemolytica and $H$. somni) in $\mathrm{BRD}$. The second limitation is the existence of individual variation, which might have contributed to variation in the results. Third, the $\mathrm{PK}$ values obtained in plasma concentration-time profiles were used for PK/PD integration and modeling. However, these drug concentrations do not accurately reflect the actual realtime concentrations in the bacterial microenvironment. Further studies should take these factors into consideration.

\section{CONCLUSION}

In the present study, a neutropenic mouse lung infection model was first used to evaluate the in vivo $\mathrm{PK}$ and $\mathrm{PD}$ indices of gamithromycin. The $f \mathrm{AUC}_{0-24} / \mathrm{MIC}$ ratio showed the strongest correlation with antibacterial activity. Furthermore, the current study suggests that the bacteriostatic action, $1-\log _{10}$ reduction, $2-\log _{10}$ reduction, and $3-\log _{10}$ reduction of the bacterial count were achieved when the $f \mathrm{AUC}_{0-24} / \mathrm{MIC}$ ratio reached 56.77, 90.18, 143.06 , and $239.44 \mathrm{~h}$, respectively. Our research provides useful pharmacological data which could assist in optimizing the clinical use of gamithromycin against infections caused by P. multocida.

\section{DATA AVAILABILITY}

The raw data supporting the conclusions of this manuscript will be made available by the authors, without undue reservation, to any qualified researcher.

\section{ETHICS STATEMENT}

This study was carried out in accordance with the recommendations of the Committee on the Ethics of Animals of the South China Agricultural University and was performed according to the American Association for Accreditation of Laboratory Animal Care guidelines. The protocol was approved by the Committee on the Ethics of Animals of the South China Agricultural University (approval number: 2017047).

\section{AUTHOR CONTRIBUTIONS}

YL and HD conceived and designed the experiments. QY, XL, and KY performed the experiments. CZ analyzed the data. QY drafted the manuscript. AC contributed to the revision. All authors read and approved the final manuscript.

\section{FUNDING}

This work was supported by the National Key Research and Development Program of China (2016YFD0501310). 


\section{REFERENCES}

Abell, K. M., Theurer, M. E., Larson, R. L., White, B. J., and Apley, M. (2017). A mixed treatment comparison meta-analysis of metaphylaxis treatments for bovine respiratory disease in beef cattle. J. Anim. Sci. 95 (2), 626-635. doi: $10.2527 /$ jas.2016.1062

Ahmad, I., Huang, L., Hao, H., Sanders, P., and Yuan, Z. (2016). Application of $\mathrm{PK} / \mathrm{PD}$ modeling in veterinary field: dose optimization and drug resistance prediction. Biomed. Res. Int. 2016 (8), 5465678. doi: 10.1155/2016/5465678

Andes, D., and Craig, W. A. (2006). Pharmacodynamics of a new cephalosporin, PPI-0903 (TAK-599), active against methicillin-resistant Staphylococcus aureus in murine thigh and lung infection models: identification of an in vivo pharmacokinetic-pharmacodynamic target. Antimicrob. Agents Chemother. 58 (4), 1376-1383. doi: 10.1128/AAC.50.4.1376-1383.2006

Baggott, D., Casartelli, A., Fraisse, F., Manavella, C., Marteau, R., Rehbein, S., et al. (2011). Demonstration of the metaphylactic use of gamithromycin against bacterial pathogens associated with bovine respiratory disease in a multicentre farm trial. Vet. Rec. 168 (9), 241-241. doi: 10.1136/vr.c6776

Berlin, S., Randow, T., Scheuch, E., Grube, M., Venner, M., and Siegmund, W. (2017). Pharmacokinetics and pulmonary distribution of gamithromycin after intravenous administration in foals. J. Vet. Pharmacol. Ther. 40 (4), 406-410. doi: $10.1111 /$ jvp.12402

Buyck, J. M., Plésiat, P., Traore, H., Vanderbist, F., Tulkens, P. M., and Van Bambeke, F. (2012). Increased susceptibility of Pseudomonas aeruginosa to macrolides and ketolides in eukaryotic cell culture media and biological fluids due to decreased expression of oprM and increased outer-membrane permeability. Clin. Infect. Dis. 55 (4), 534-542. doi: 10.1093/cid/cis473

Dabo, S. M., Taylor, J. D., and Confer, A. W. (2007). Pasteurella multocida and bovine respiratory disease. Anim. Health. Res. Rev. 8 (2), 129-150. doi: $10.1017 /$ S1466252307001399

Dedonder, K. D., Apley, M. D., Li, M., Gehring, R., Harhay, D. M., Lubbers, B. V., et al. (2015). Pharmacokinetics and pharmacodynamics of gamithromycin in pulmonary epithelial lining fluid in naturally occurring bovine respiratory disease in multisource commingled feedlot cattle. J. Vet. Pharmacol. Ther. 39 (2), 157-166. doi: 10.1111/jvp.12267

Ferran, A. A., Toutain, P. L., and Bousquet-Mélou, A. (2011). Impact of early versus later fluoroquinolone treatment on the clinical; microbiological and resistance outcomes in a mouse-lung model of Pasteurella multocida infection. Vet. Microbiol. 148 (2), 292-297. doi: 10.1016/j.vetmic.2010.09.005

Forbes, A. B., Ramage, C., Sales, J., Baggott, D., and Donachie, W. (2011). Determination of the duration of antibacterial efficacy following administration of gamithromycin using a bovine Mannheimia haemolytica challenge model. Antimicrob. Agents Chemother. 55 (2), 831-835. doi: 10.1128/ AAC.00552-10

Griffin, D., Chengappa, M. M., Kuszak, J., and Mcvey, D. S. (2010). Bacterial pathogens of the bovine respiratory disease complex. Vet. Clin. N. Am-Small 26 (2), 381-394. doi: 10.1016/j.cvfa.2010.04.004

Hoffman, H. L., Klepser, M. E., Ernst, E. J., Petzold, C. R., Sa'adah, L. M., and Doern, G. V. (2003). Influence of macrolide susceptibility on efficacies of clarithromycin and azithromycin against Streptococcus pneumoniae in a murine lung infection model. Antimicrob. Agents Chemother. 47 (2), 739-746. doi: 10.1128/AAC.47.2.739-746.2003

Huang, R. A., Letendre, L. T., Banav, N., Fischer, J., and Somerville, B. (2010). Pharmacokinetics of gamithromycin in cattle with comparison of plasma and lung tissue concentrations and plasma antibacterial activity. J. Vet. Pharmacol. Ther. 33 (3), 227-237. doi: 10.1111/j.1365-2885.2009.01125.x

Jones, C., and Chowdhury, S. (2007). A review of the biology of bovine herpesvirus type $1(\mathrm{BHV}-1)$, its role as a cofactor in the bovine respiratory disease complex and development of improved vaccines. Anim. Health. Res. Rev. 8 (2), 187-205. doi: 10.1017/S146625230700134X

Jones, M. L., Washburn, K. E., Fajt, V. R., Rice, S., and Coetzee, J. F. (2015). Synovial fluid pharmacokinetics of tulathromycin, gamithromycin and florfenicol after a single subcutaneous dose in cattle. BMC Vet. Res. 11 (1), 1-8. doi: 10.1186/ s12917-015-0346-4

Kellermann, M., Huang, R. A., Forbes, A. B., and Rehbein, S. (2014). Gamithromycin plasma and skin pharmacokinetics in sheep. Res. Vet. Sci. 97 (2), 199-203. doi: 10.1016/j.rvsc.2014.08.008
Lees, P., Concordet, D., Toutain, P. L., and Aliabadi, F. S., (2006). Drug selection and optimization of dosage schedules to minimize antimicrobial resistance. Washington, DC: ASM Press, 49-72.

Mitchell, J. D., Shan, G., Mckellar, Q. A., and Mckeever, D. J. (2013). In vitro pharmacodynamics of gamithromycin against Mycoplasma mycoides subspecies mycoides small colony. Vet. J. 197 (3), 806-811. doi: 10.1016/j.tvjl.2013.05.025

Muraro, M., Boso, M., Basano, F. S., Nazzari, R., Bassini, A., and Soriolo, A. (2012). Comparative efficacy of two antimicrobials when used for the prevention of bovine respiratory disease (BRD), in two farms with different management of arrival conditioning. Large Anim. Rev. 18 (6), 283-290. doi: 10.2527/ jas.2012-5460

Mustafa, M. H., Khandekar, S., Tunney, M. M., Elborn, J. S., Kahl, B. C., Denis, O., et al. (2017). Acquired resistance to macrolides in Pseudomonas aeruginosa from cystic fibrosis patients. Eur. Respir. J. 49, 1601847. doi: 10.1183/13993003.01847-2016

O'Connor, A. M., Yuan, C., Cullen, J. N., Coetzee, J. F., Da, S. N., and Wang, C. (2016). A mixed treatment meta-analysis of antibiotic treatment options for bovine respiratory disease - an update. Prev. Vet. Med. 132, 130-139. doi: 10.1016/j.prevetmed.2016.07.003

Portis, E., Lindeman, C., Johansen, L., and Stoltman, G. (2012). A ten-year (2000-2009) study of antimicrobial susceptibility of bacteria that cause bovine respiratory disease complex-Mannheimia haemolytica, Pasteurella multocida, and Histophilus somni-in the United States and Canada. J. Vet. Diagn. Invest. 24 (5), 932-944. doi: 10.1177/1040638712457559

Qu, Y., Qiu, Z., Cao, C., Lu, Y., Sun, M., Liang, C., et al. (2015). Pharmacokinetics/ pharmacodynamics of marbofloxacin in a Pasteurella multocida serious murine lung infection model. BMC Vet. Res. 11 (1), 294. doi: 10.1186/ s12917-015-0608-1

Rossi, C. A. S., Vandoni, S. L., Bonfanti, M., and Forbes, A. B. (2010). Effects of arrival medication with gamithromycin on bovine respiratory disease in feedlot cattle in Italy. Int. J. Appl. Res. Vet. M. 8 (2), 87-96. doi: 10.1136/ inp.b5495

Snowder, G. D., Van Vleck, L. D., Cundiff, L. V., and Bennett, G. L. (2006). Bovine respiratory disease in feedlot cattle: environmental, genetic, and economic factors. J. Anim. Sci. 84 (8), 1999-2008. doi: 10.2527/jas.2006-046

Snowder, G. D., Van Vleck, L. D., Cundiff, L. V., Bennett, G. L., Koohmaraie, M., and Dikeman, M. E. (2007). Bovine respiratory disease in feedlot cattle: phenotypic, environmental, and genetic correlations with growth, carcass, and longissimus muscle palatability traits. J. Anim. Sci. 85 (8), 1885-1892. doi: $10.2527 /$ jas.2007-0008

Snyder, E., Credille, B., Berghaus, R., and Giguère, S. (2017). Prevalence of multi drug antimicrobial resistance in isolated from high-risk stocker cattle at arrival and two weeks after processing. J. Anim. Sci. 95 (3), 1124. doi: 10.2527/ jas.2016.1110

So, W., Crandon, J. L., and Nicolau, D. P. (2015). Pharmacodynamic profile of GSK2140944 against methicillin-resistant Staphylococcus aureus in a murine lung infection model. Antimicrob. Agents Chemother. 59 (8), 4956-4961. doi: 10.1128/AAC.00625-15

Tessier, P. R., Kim, M. K., Zhou, W., Xuan, D., Li, C., Ye, M., et al. (2002). Pharmacodynamic assessment of clarithromycin in a murine model of pneumococcal pneumonia. Antimicrob. Agents Chemother. 46 (5), 1425-1434. doi: 10.1128/AAC.46.5.1425-1434.2002

Thabit, A. K., Crandon, J. L., and Nicolau, D. P. (2016). Pharmacodynamic and pharmacokinetic profiling of delafloxacin in a murine lung model against community-acquired respiratory tract pathogens. Int. J. Antimicrob. Agents 48 (5), 535-541. doi: 10.1016/j.ijantimicag.2016.08.012

Toutain, P. L., del Castillo, J. R., and Bousquetmélou, A. (2002). The pharmacokinetic-pharmacodynamic approach to a rational dosage regimen for antibiotics. Res. Vet. Sci. 73 (2), 105-114. doi: 10.1016/S0034-5288(02)00039-5

Toutain, P. L., Potter, T., Pelligand, L., Lacroix, M., Illambas, J., and Lees, P. (2017) Standard PK/PD concepts can be applied to determine a dosage regimen for a macrolide: the case of tulathromycin in the calf. J. Vet. Pharmacol. Ther. 40, 16-27. doi: 10.1111/jvp.12333

Watteyn, A., Plessers, E., Wyns, H., De, B. S., De, B. P., and Croubels, S. (2013). Pharmacokinetics of gamithromycin after intravenous and subcutaneous administration in broiler chickens. Poult. Sci. 92 (6), 1516-1522. doi: 10.3382/ ps.2012-02932 
Watteyn, A., Devreese, M., Siegrid, D. B., Wyns, H., Plessers, E., and Boyen, F. (2015). Pharmacokinetic and pharmacodynamic properties of gamithromycin in turkey poults with respect to Ornithobacterium rhinotracheale. Poult. Sci. 94, 2066-2074. doi: 10.3382/ps/pev217

Wyns, H., Meyer, E., Plessers, E., Watteyn, A., De, B. S., De, B. P., et al. (2014). Pharmacokinetics of gamithromycin after intravenous and subcutaneous administration in pigs. Res. Vet. Sci. 96 (1), 160-163. doi: 10.1016/j. rvsc.2013.11.012

Zeng, D., Sun, M., Lin, Z., Li, M., Gehring, R., and Zeng, Z. (2018). Pharmacokinetics and pharmacodynamics of tildipirosin against Pasteurella multocida in a murine lung infection model. Front. Microbiol. 9, 1038. doi: $10.3389 /$ fmicb. 2018.01038

Zhou, Q., Zhang, G., Wang, Q., Liu, W., Huang, Y., Yu, P., et al. (2017a). Pharmacokinetic/pharmacodynamic modeling of tulathromycin against Pasteurella multocida in a porcine tissue cage model. Front. Pharmacol. 8, 392. doi: 10.3389/fphar.2017.00392
Zhou, Y. F., Tao, M. T., Huo, W., Liao, X. P., Sun, J., and Liu, Y. H. (2017b). In vivo pharmacokinetic and pharmacodynamic profiles of antofloxacin against Klebsiella pneumoniae in a neutropenic murine lung infection model. Antimicrob. Agents Chemother. 61 (5), AAC.02691-02616. doi: 10.1128/AAC.02691-16

Conflict of Interest Statement: The authors declare that the research was conducted in the absence of any commercial or financial relationships that could be construed as a potential conflict of interest.

Copyright (c) 2019 Yang, Liu, Zhang, Yong, Clifton, Ding and Liu. This is an openaccess article distributed under the terms of the Creative Commons Attribution License (CC BY). The use, distribution or reproduction in other forums is permitted, provided the original author(s) and the copyright owner(s) are credited and that the original publication in this journal is cited, in accordance with accepted academic practice. No use, distribution or reproduction is permitted which does not comply with these terms. 